University of Nebraska - Lincoln

DigitalCommons@University of Nebraska - Lincoln

2011

Moisture-Induced TBC Spallation on Turbine Blade Samples

James L. Smialek

NASA Glenn Research Center, James.L.Smialek@grc.nasa.gov

Follow this and additional works at: https://digitalcommons.unl.edu/nasapub

Part of the Physical Sciences and Mathematics Commons

Smialek, James L., "Moisture-Induced TBC Spallation on Turbine Blade Samples" (2011). NASA

Publications. 90.

https://digitalcommons.unl.edu/nasapub/90

This Article is brought to you for free and open access by the National Aeronautics and Space Administration at DigitalCommons@University of Nebraska - Lincoln. It has been accepted for inclusion in NASA Publications by an authorized administrator of DigitalCommons@University of Nebraska - Lincoln. 


\title{
Moisture-Induced TBC Spallation on Turbine Blade Samples
}

\author{
James L. Smialek* \\ NASA Glenn Research Center, United States
}

\section{A R T I C L E I N F O}

Available online 22 July 2011

\section{Keywords:}

TBC

alumina scales

moisture

hydrogen

oxide-metal adhesion

\begin{abstract}
A B S T R A C T
Delayed failure of TBCs is a widely observed laboratory phenomenon, although many of the early observations went unreported. "The weekend effect" or "DeskTop Spallation" (DTS) is characterized by initial survival of a TBC after accelerated laboratory thermal cycling, followed by failure when exposed to ambient humidity or water. Once initiated, failure can progress to completion quite dramatically in less than a second. To this end, the water drop test and digital video recordings have become useful techniques in studies at NASA (Smialek, Zhu, Cuy), DECHMA (Rudolphi, Renusch, Schütze), and CNRS Toulouse/SNECMA (Déneux, Cadoret, Hervier, Monceau). In the present study the results for a commercial turbine blade, with a standard EB-PVD 7YSZ TBC top coat and Pt-aluminide diffusion bond coat are reported. Cut sections were intermittently oxidized at $1100^{\circ}, 1150^{\circ}$, and $1200^{\circ} \mathrm{C}$ and monitored by weight change and visual appearance. Failures were distributed widely over a 5-100 hr time range, decreasing with oxidation temperature. At some opportune times, failure was captured by video recording, documenting the appearance and speed of the moisture-induced spallation process. The failure interfaces exhibited, on the TBC side, alumina scale grains (decorated with Ta-rich oxide particles) and, on the exposed bare metal surface of the blade, imprints from the scale grains (with alumina islands and streamers). The phenomenon is based on moisture-induced delamination at the alumina scale bond coat interface. Cycling damage is a contributing factor as cracking allows access of moisture to this interface, while high strain energy provides the driving force for spallation. It has been proposed that moisture reacts with aluminum in the bond coat and releases hydrogen atoms that 'embrittle' the interface. A modified chemical viewpoint of scale adhesion results, including a negative synergistic effect with interfacial sulfur.
\end{abstract}

Published by Elsevier B.V.

\section{Introduction and background}

Thermal barrier coatings (TBC), widely used on metallic combustor liners and airfoils in turbine engines, may suffer degradation from surface sintering, phase transformations, cyclic damage, bond coat oxidation and rumpling, calcium magnesium aluminosilicate (CMAS) and salt deposits, to name a few. Regarding bond coat oxidation, it is well established that the integrity of a thin, adherent, slow-growing alumina scale at the TBC-bond coat interface is required for optimum performance. Specifically, any factor that encourages scale spallation may ultimately trigger TBC debonding and failure. Accordingly, beneficial noble metal, reactive element, and detrimental sulfur impurity effects on scale growth and adhesion to the bond coat become of paramount importance.

In addition, moisture has often been found to be a negative factor in laboratory tests regarding alumina scale adhesion and TBC failure. That is, scales and TBCs that were otherwise adherent in normal furnace cycling were found to fail when exposed to ambient moisture contents, well after cool down to room temperature. This is not to say that young adherent alumina scales are subject to moisture effects. Indeed, the detrimental effects only arise for at-risk or mature (thick and heavily

\footnotetext{
* Tel.: +1 216-433-5500.

E-mail address: James.L.Smialek@grc.nasa.gov.
}

cycled) scales. There is a continuum of degradation factors that come in play for the effect to be manifested: moisture effects become more pronounced as the scales are subjected to greater strain energy and cyclic damage, i.e., have some cracking or spalling. Simple diffusion aluminide bond coats, with no Pt or reactive elements, do not have optimum scale adhesion and are subject to the detrimental effects of sulfur interfacial segregation and voiding. The above factors increase the probability of some interfacial spalling and access of ambient humidity to exposed metal. It is this situation that is ripe for Moisture-Induced Delayed Spallation (MIDS).

Indeed a 'sweet zone' for MIDS can be conceptually described at a given level of adhesion. It is determined by sulfur and/or Pt, Y, Hf, etc. dopant content and broadens with moisture level, time of exposure, strain energy, and cyclic damage, Fig. 1. In Fig. 1a, the amount of retained scale after cool down is shown schematically as a sigmoidal curve, increasing as sulfur content decreases from critical contents of about 1 down to $0.1 \mathrm{ppmw}$. (The case shown would apply roughly to an undoped alloy). The amount of retained scale after moisture exposure is shown as another sigmoidal curve (for the undoped case), but below that of the as-cooled curve. Thus, the observed, net moisture effect is the difference between the two, Fig. $1 \mathrm{~b}$, and is a maximum at some intermediate sulfur (or Pt, Y, Hf) level. At higher sulfur levels, the scale spalls initially on cooldown before any moisture exposure, at lower levels the scale remains adherent even after moisture exposure. The 
a

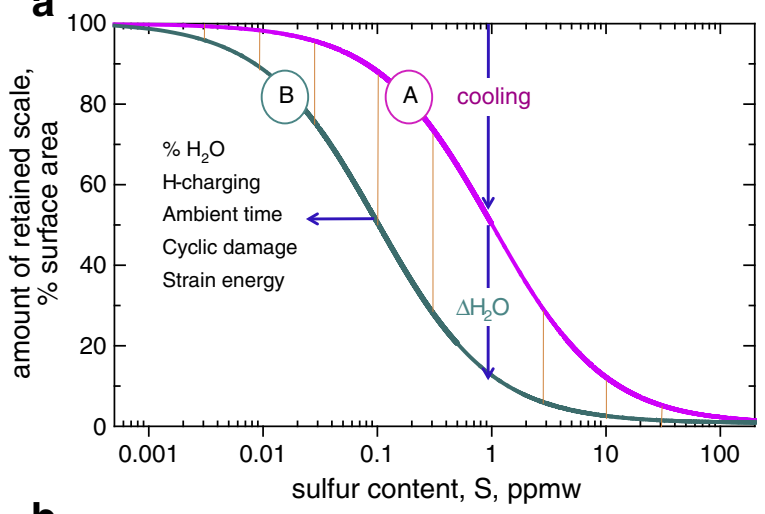

b

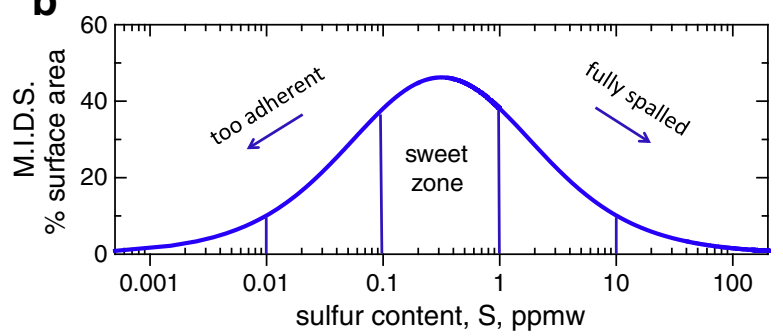

Fig. 1. Schematic sigmoidal trends in retained scale after cooling and moisture exposure as a function of sulfur content: a) Shift in final retained scale to lower sulfur levels due to moisture factors. b) Net amount of moisture-induced spallation is best manifested at an intermediate level of adhesion.

shiftable nature of the 'sweet zone' accounts for the rather enigmatic nature of the phenomenon. These characteristics and an extensive defense of a possible mechanism were presented in a recent position paper [1]. There it was postulated that moisture can react with aluminum in the bond coat to form a hydroxide molecule, releasing hydrogen into the strained interface and degrading adhesion. A number of observations from tangential fields are presented in defense of this hypothesis. Indeed, supporting cathodic hydrogen charging experiments were shown to produce interfacial alumina scale spallation for mature cycled scales on Rene N5 + Y [2,3].

Regarding TBC phenomena more specifically, an early indication of a moisture effect was the observation of slow delamination (blister) growth in a physical vapor deposited (PVD) TBC as detected by photoluminescence (piezospectroscopy) of the underlying alumina scale [4]. Studies of oxidized air plasma spray (APS) TBCs on no-bond coat superalloys were found to completely detach unexpectedly, well after cool down, but especially upon water immersion in $1100^{\circ}$ and $1150{ }^{\circ} \mathrm{C}$ tests $[5,6]$. Video recordings were later used to demonstrate water drop induced spallation of a PVD TBC from a Pt-aluminide bond coat after $300 \mathrm{hr}$ cyclic oxidation at $1150{ }^{\circ} \mathrm{C}$ [7] . The water drop video test, combined with acoustic emission, revealed cracking history and PVD TBC delamination from a vacuum plasma spray (VPS) NiCoCrAlY bond coat that was oxidized isothermally for $310 \mathrm{hr}$ at $1050{ }^{\circ} \mathrm{C}[8,9]$. In addition, PVD TBCs on Pt-aluminide bond coats failed above $170 \mathrm{hr}$ when exposed to ambient humidity or water [10]. However, they were shown to survive 2001 -hr cycles at $1150{ }^{\circ} \mathrm{C}$ when stored in dry atmospheres indefinitely. This suggests that moisture may sometimes be a necessary factor for spallation, though it is not sufficient. Many of these studies also show that moisture encourages TBC spallation by decreasing adhesion of the alumina scale to the metal bond coat. That is to say, DTS and MIDS are rooted in the same sensitivity of the oxide metal interface to moisture.

While confirmed in the laboratory, the same moisture phenomenon has not been observed in the field or in service. Component and engine design avoid severe bond coat oxidation and preclude the high strain energy needed to sensitize the interface to hydrogen or whatever the root cause may be. Given this background, the purpose of the present study was to demonstrate, by accelerated laboratory exposures, the water test on similar coated commercial hardware samples. These were sectioned from an actual engine blade pulled from service. Failure modes were noted at various intervals for $1100^{\circ}$, $1150^{\circ}$, and $1200^{\circ} \mathrm{C}$ intermittent oxidation tests. On occasion, the TBC spalling event was able to be captured on video. The resulting failure surfaces are characterized by optical and scanning electron microscopy (SEM), including backscatter electron (BSE) imaging and energy dispersive spectroscopy (EDS).

\section{Experimental}

A first stage turbine blade was obtained from a prominent company. It had been removed from service in an aero-derivative ground power installation after considerable use, the details being proprietary. The blade was a directionally solidified Ni-base superalloy. The TBC was a commercial EB-PVD 7YSZ TBC using a Ni(Pt)Al chemically vapor deposited (CVD) platinum aluminide diffusion coating. These bond coatings typically employ $5-10 \mu \mathrm{m}$ of electroplated Pt, followed by aluminizing to produce $20-40 \mu \mathrm{m}$ of beta-phase $\mathrm{Ni}(\mathrm{Pt}) \mathrm{Al}$. The substrate alloy (confidential), is seen to contain $\sim 5$ ppmw $S$ impurity from nominally similar processing. An Hf dopant was also present in the substrate, but no Y. The blade was sectioned by electro-discharge machining into three $\sim 1 \mathrm{~cm}$ high chord slices (\#A, B, C) which were then sectioned into six $\sim 1 \mathrm{~cm}$ wide specimens (\#1, 2, 3, $4,5,6)$. All specimens had cut edges polished with SiC emery to a 600 grit finish. The TBC appeared to be of uniform cross-section before testing, however no metallographic measurements were obtained before oxidation. The samples were ultrasonically cleaned in detergent, then in alcohol and dried. Examples of the sliced specimens, from chords B and C, are shown in Fig. 2. The sectioned samples are labeled 1 through 6 starting at the leading edge as shown. Some sections $(\# 1,5,6)$ present larger amounts of TBC coated surface on both pressure (concave) and suction (convex) sides. Others have essentially one major TBC surface due to the blade curvature. It can be seen that the samples expose many internal surfaces as well as the cut edges. Both of these issues will complicate the interpretation of oxidative weight changes, especially at higher temperatures. Also, weight changes are reported in terms of $\mathrm{mg}$ and not $\mathrm{mg} / \mathrm{cm}^{2}$ for the

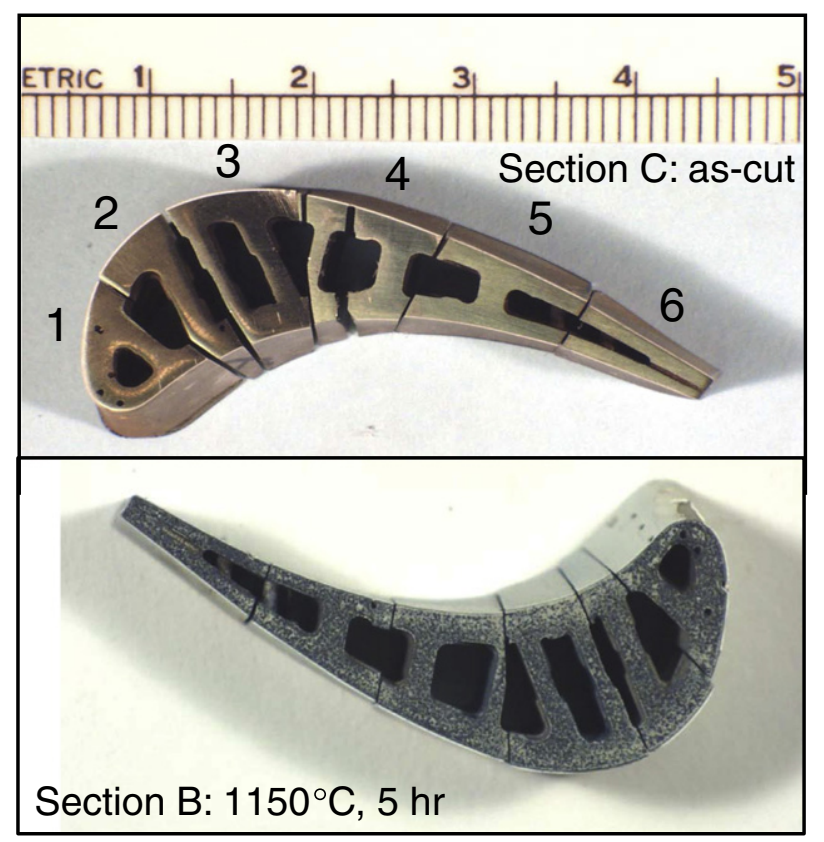

Fig. 2. Sliced TBC-coated turbine blade chord and six test sections for water drop experiments. Section C, as-cut and sanded, and Section B, after oxidation at $1150{ }^{\circ} \mathrm{C}$ for $5 \mathrm{hr}$. 
same reasons. Despite these shortcomings, weight change can be a useful measure of TBC spallation, especially at the lower temperatures. For the most part, the percentage of TBC lost was complete and corresponded to massive weight drops that occurred after cooldown. Thus monitoring TBC spallation by image analysis would not necessarily provide additional value.

Samples were placed in a high purity alumina boat, oxidized intermittently at $1100^{\circ}, 1150^{\circ}$, or $1200^{\circ} \mathrm{C}$ in a box furnace for the slices named A, B, and C, respectively. Various heating intervals were used, generally following the progressive schedule of $1,2,5,10,15, \ldots$ $50,75,100 \mathrm{hr}$, total time. Samples were weighed to $\pm 0.05 \mathrm{mg}$ after each cooldown. The weights were corrected for any balance drift by frequently weighing the same calibrated $3.00000 \mathrm{~g}$ standard weight. With increased oxidation time, some samples produced TBC spallation on cooldown. Other samples were occasionally exposed to a water drop from a wash bottle, sometimes monitored by digital video recording. Also, some samples were subjected to sequential immersions in acetone, alcohol, and water and re-weighed in order to isolate any effects of water from other 'dry' liquids. Failed TBC interfaces were examined by optical microscopy and SEM/EDS.

\section{Results}

Prior to oxidation, an as-received blade was exposed to water immersion and exhibited no effect. Attempts to debond by cathodic hydrogen charging also exhibited no effect. The moisture effect was only produced by subjecting the samples to accelerated oxidation (below), with partially exposed scale-metal interfaces (at the cut edges).

\section{1. $1100^{\circ} \mathrm{C}$ intermittent oxidation}

The weight change with $1100{ }^{\circ} \mathrm{C}$ oxidation time is shown for chord A samples in Fig. 3. Here the test begins with most samples producing a small weight gain on the order of $1 \mathrm{mg}$ in the first $10 \mathrm{hr}$. Eventually, weight loss occurred at an average rate (dashed line) of about $0.15 \mathrm{mg} / \mathrm{hr}$ after $10 \mathrm{hr}$, just due to oxidative issues with the cut and internal surfaces of the blade alloy. Additional drops in weight, often quite abrupt, occurred with partial TBC coating loss on one (concave or convex) surface of a sample. Failure occurred by both normal cool down stresses or by the water drop test, with some sample to sample variation. (The water drop failures are indicated by the star-point symbols). Sample A1 exhibited a small amount of bare metal spallation at the leading edge after cool down from $40 \mathrm{hr}$ of oxidation. Sequential immersions, up to $15 \mathrm{~min}$. each, in acetone, alcohol, and water were performed after 10-75 hr test times. Acetone and alcohol never caused TBC spallation, only some small weight losses due to

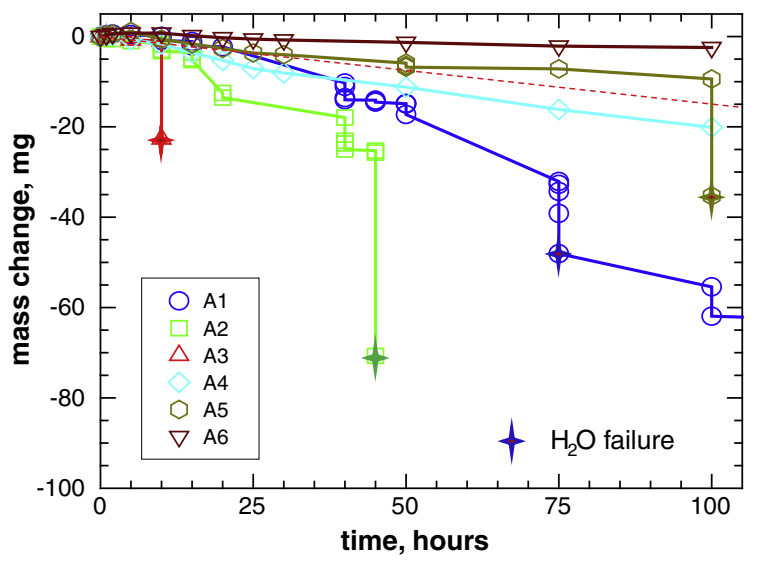

Fig. 3. Mass losses for $1100{ }^{\circ} \mathrm{C}$ intermittent oxidation of Section A samples. Water drop spallation failures indicated by star symbol. Dashed line indicates approximate loss rate for exposed superalloy cut edges and internal blade surfaces. spallation of oxides at the cut superalloy surfaces. At $75 \mathrm{hr}$, TBC spallation on one side occurred by cooling and on the other by subsequent water exposure. Sample A2 exhibited partial delamination early in the test, but the TBC remained loosely attached. It was subjected to the same sequential immersions as A1, again with little effect of acetone or alcohol. Some loose TBC segments chipped off at 20 and $40 \mathrm{hr}$ just due to handling. Sample A3 lost one surface of the TBC due to water immersion after just $10 \mathrm{hr}$ of oxidation, and no further testing was done. Samples A4, A5, and A6 were relatively unaffected for the first $50 \mathrm{hr}$. Thus moisture induced TBC failures can be seen for samples A3, A2, A1, and A5 occurring at 10, 45, 75, and $100 \mathrm{hr}$. total oxidation time, respectively.

It is also important to acknowledge when water drop testing did not result in TBC failure and how those times relate to normal cooling failures, if any. (It goes without saying that any moisture-induced failure implies that the coating had survived cooldown, i.e., falls in the metastable 'sweet zone'). Accordingly, a summary of the failure history of each sample is presented in the bar chart of Fig. 4. Generally double bars are presented and correspond to the TBC on two sides of the sample. Here it can be seen that one TBC side of sample A1 exhibited cooling spallation and the other side water failure, both at $75 \mathrm{hr}$. Sample A2 survived moisture testing at $40 \mathrm{hr}$., then failed on immersion at $45 \mathrm{hr}$. and was pulled from further testing. Sample A3 survived immersion at $5 \mathrm{hr}$, then failed at just $10 \mathrm{hr}$, and this sample was also pulled. Sample A5 survived the water drop test at 50 and $75 \mathrm{hr}$, then failed with a water drop on one side at $100 \mathrm{hr}$. Finally, samples A4 and A6 survived cooldown up to $100 \mathrm{hr}$ oxidation and are being maintained in a drying oven for a subsequent water test and video.

\section{2. $1150^{\circ} \mathrm{C}$ intermittent oxidation}

The weight change response for testing samples from chord B at $1150{ }^{\circ} \mathrm{C}$ is shown in Fig. 5. Here about $10 \mathrm{mg}$ weight loss was occurring after $20 \mathrm{hr}$ just due to blade alloy oxidation, i.e., at an average rate of $0.5 \mathrm{mg} / \mathrm{hr}$. An additional $50 \mathrm{mg}$ or so drop in weight, often quite abrupt, was generally associated with coating failure on one major surface of a sample. Moisture induced failures can be seen for samples $1-4$, occurring over 12.5 to $35 \mathrm{hr}$ total test time. A summary of the survival/failure history of each sample is presented in Fig. 6. Here it can be seen that, after $12.5 \mathrm{hr}$ oxidation, sample B1 exhibited cooling spallation on one side and subsequent water failure on the other. One side of sample B2 survived moisture testing at $10 \mathrm{hr}$, then it failed on both sides at $15 \mathrm{hr}$. Sample B3 showed partial failure on cooldown and more with moisture exposure after $15 \mathrm{hr}$, then completed spallation by moisture failure at $16 \mathrm{hr}$. Sample B4 survived the water drop test at $20 \mathrm{hr}$, then partially failed at $22.5 \mathrm{hr}$. The remnant coating survived moisture exposure at $30 \mathrm{hr}$, then failed at $35 \mathrm{hr}$. Finally,

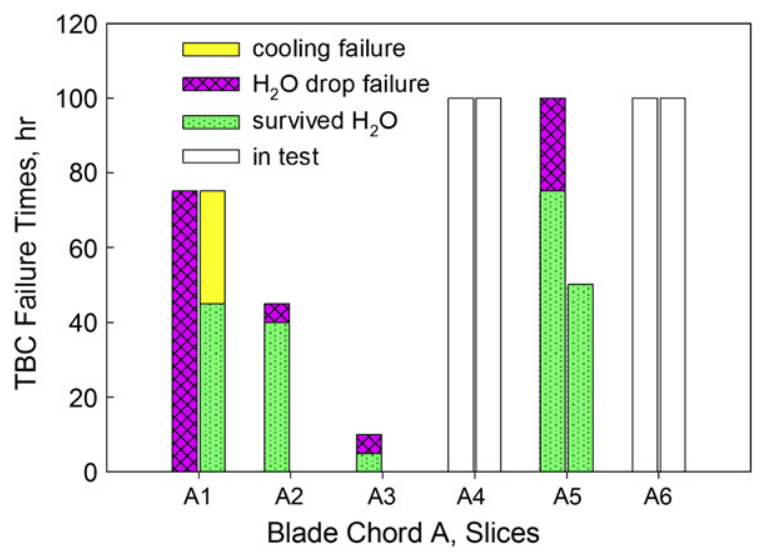

Fig. 4. Survival/failure history for TBC spallation from cool down and water drop tests. Section $\mathrm{A}, 1100^{\circ} \mathrm{C}$ intermittent oxidation. $100 \mathrm{hr}$ maximum failure time for water drop (so far). 


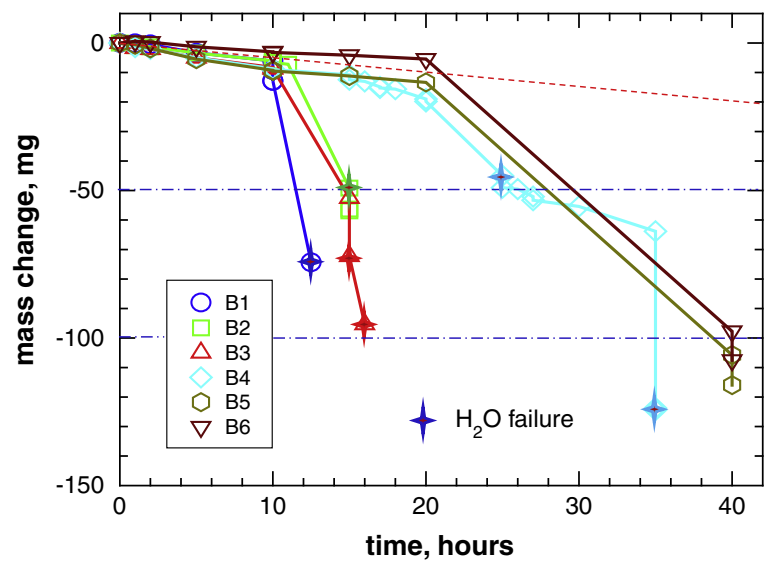

Fig. 5. Mass losses for $1150{ }^{\circ} \mathrm{C}$ intermittent oxidation of Section B samples. Water drop spallation failures indicated by star symbol. Dashed line indicates approximate loss rate for exposed superalloy cut edges and internal blade surfaces. Horizontal dot/dash lines indicate approximate magnitude of mass loss due to TBC spallation from one or both major surfaces.

both sides of samples B5 and B6 both survived cooldown and the water drop test at $20 \mathrm{hr}$, then failed on cooldown at $40 \mathrm{hr}$ (due to the final long $20 \mathrm{hr}$ interval with no intermediate inspection interval).

\section{3. $1200^{\circ} \mathrm{C}$ intermittent oxidation}

The weight change response for testing samples from chord $\mathrm{C}$ at $1200{ }^{\circ} \mathrm{C}$ is shown in Fig. 7. Now the oxidation of the base metal produced nearly $50 \mathrm{mg}$ weight loss after just 5-10 hr oxidation, or at an average rate of $\sim 7 \mathrm{mg} / \mathrm{hr}$. In many cases a weight was not obtained after coodown until the water drop test was applied along with the attempt of obtaining a failure video. This strategy, combined with large oxidative losses, obscured the usual abrupt drops in weight associated with coating failure. Nevertheless, moisture-induced TBC failures are noted for samples C1, C2, C4, and C6 occurring at 10, 5, 15, and $20 \mathrm{hr}$ total test time, respectively. A summary of the survival/failure history is presented in Fig. 8. Here it can be seen that both sides of sample C1 spalled exclusively by water failure at just $10 \mathrm{hr}$. Sample C2 spalled partially by cooldown, then by moisture testing, both at $10 \mathrm{hr}$. Samples C3-C6 exhibited partial failure on cooldown from 10-20 hr, survived moisture testing for 5-15 hr, and failed in water drop tests at 15 and $20 \mathrm{hr}$. At this temperature, the overlap of the various survival/failure times were more prevalent, as the lifetimes became highly compressed by oxidative degradation.

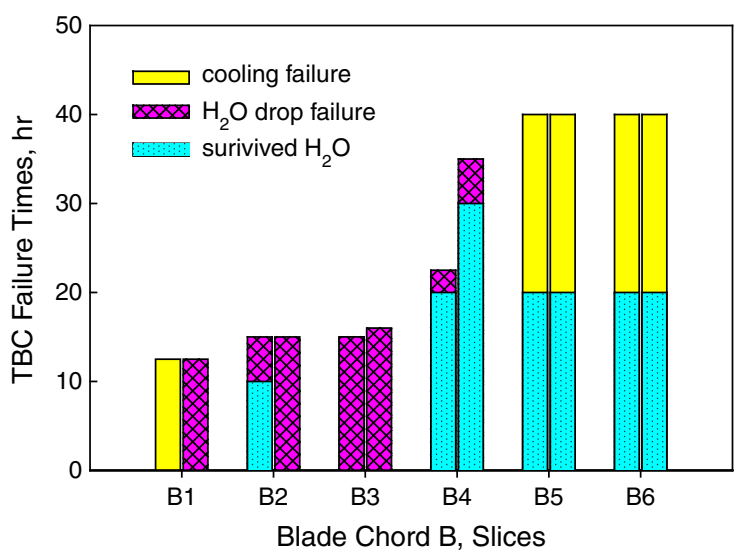

Fig. 6. Survival/failure history for TBC spallation from cool down and water drop tests. Section $\mathrm{B}, 1150{ }^{\circ} \mathrm{C}$ intermittent oxidation. $35 \mathrm{hr}$ maximum failure time for water drop.

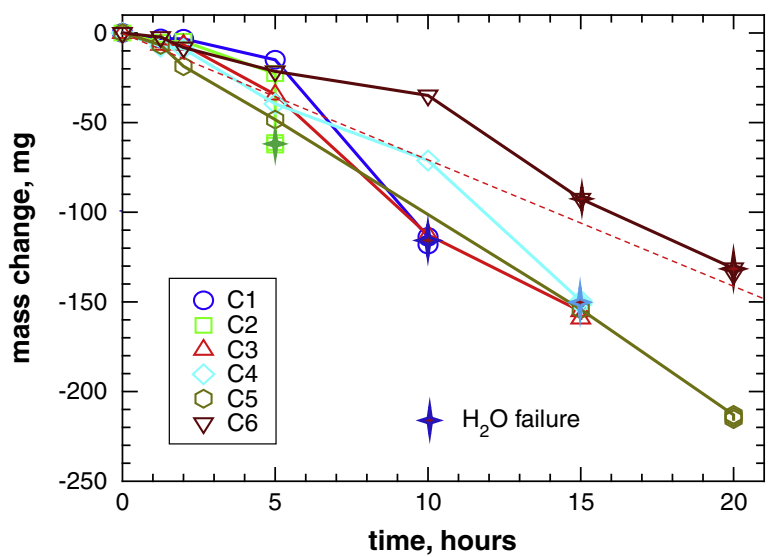

Fig. 7. Mass losses for $1200{ }^{\circ} \mathrm{C}$ intermittent oxidation of Section C samples. Water drop spallation failures indicated by star symbol. Dashed line indicates approximate loss rate for uncoated superalloy cut edges.

A summary of the average survival/failure history is shown in Fig. 9. Here the water drop failure is shown to decrease substantially from $60 \mathrm{hr}$ at $1100{ }^{\circ} \mathrm{C}$ to $12 \mathrm{hr}$ at $1200^{\circ} \mathrm{C}$. (Two samples have not failed at $100 \mathrm{hr}$ for $1100{ }^{\circ} \mathrm{C}$ tests. This will most likely increase all values at $1100^{\circ} \mathrm{C}$.) The failure times by simple cooling follow the same trend and are often within the standard deviation of the water drop failure times. This emphasizes the experimental difficulty in demonstrating failure by water drop video recording.

\subsection{Optical macro, microphotos of visual appearance and video recording of spallation}

The appearance of a cut surface of sample B2, oxidized at $1150{ }^{\circ} \mathrm{C}$ for $5 \mathrm{hr}$ is shown in the macrograph of Fig. 10. The TBC is seen to be intact as the white top layer. It is attached to the $\mathrm{Ni}(\mathrm{Pt}) \mathrm{Al}$ bond coat which exhibits only modest oxidation. The exposed substrate however shows a preponderance of blue $\left(\mathrm{NiAl}_{2} \mathrm{O}_{4}\right.$ spinel) scale. (Blue $\mathrm{NiAl}_{2} \mathrm{O}_{4}$ spinels are typical degradation stages of $\mathrm{Ni}$-base superalloys) [ref. 12]. Spalling to (reflective) bare metal and large grain NiO nodules (dark) can be seen in local areas (arrows). These substrate features account for the majority of the oxidative weight losses discussed above that were not associated with TBC spallation.

The water test sometimes results in complete delamination and removal of the TBC in large intact pieces. Such a result is shown in Fig. 11 for sample $\mathrm{C} 2$ that failed by immersion in water for 2 min. after $5 \mathrm{hr}$ oxidation at $1200^{\circ} \mathrm{C}$. The single TBC flake is positioned against the surface it delaminated from, but flipped inside out for a definitive

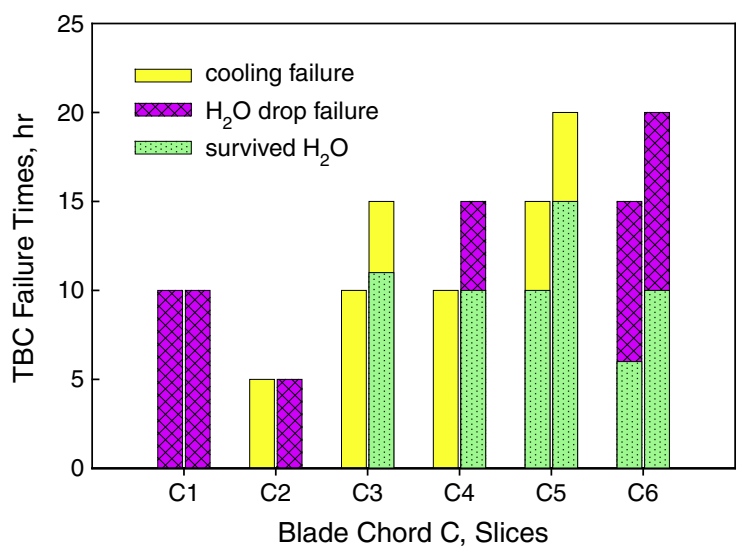

Fig. 8. Survival/failure history for TBC spallation from cool down and water drop tests. Section C, $1200{ }^{\circ} \mathrm{C}$ intermittent oxidation. $20 \mathrm{hr}$ maximum failure time for water drop. 


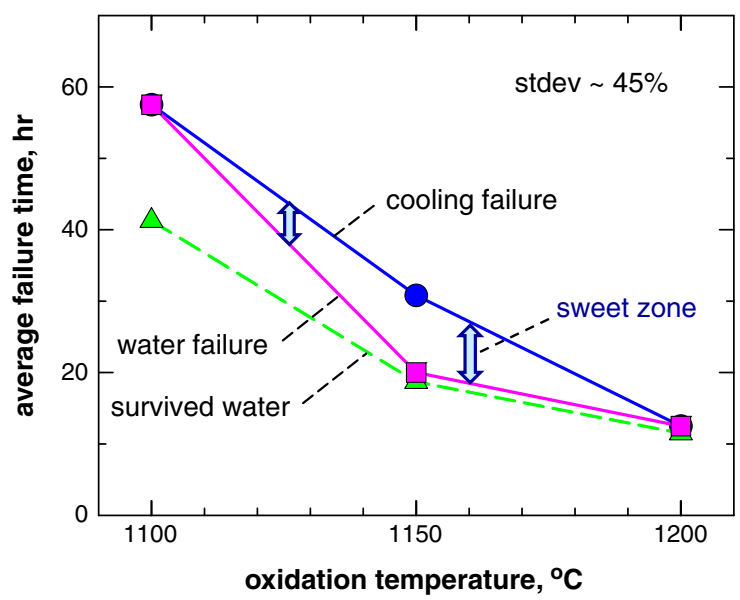

Fig. 9. Effect of oxidation temperature on average TBC failure times (on cool down, with water drop, or survived).

illustration. The exposed metal (bond coat) side is seen to be relatively free of TBC. Failure at the scale-metal interface was typical both for cooling failures and water-induced failures.

Another typical appearance of a tested and failed sample is shown in Fig. 12. Here some TBC is shown damaged and partially detached. The exposed bond coat is also apparent as reflective metal, with little evidence of retained scale. (The edges of the sample had bond coat chipping damage from cutting, allowing substrate oxidation).

Often, the TBC was observed to delaminate in very small fragments in a more or less sequential fashion, progressing from a single failure point, generally near a cut edge. A video sequence of such a progression was obtained for a number of samples when they were exposed to a squirt of water from a wash bottle. Still frames captured from such a video are presented in Fig. 13 for sample C6 oxidized at $1200{ }^{\circ} \mathrm{C}$ for $15 \mathrm{hr}$. The first frame (a) shows the intact sample as the water stream is applied from the upper right. The superimposed time code bar is in hr:min:sec; $1 / 30$ sec., indexed from the start of the video. The next frame (b) shows that failure initiated at the upper right edge (arrow) after about 22 seconds of water exposure. Then the rapid progression of delamination is seen in the subsequent frames, spanning about $1 \mathrm{sec}$. additional cumulative time expired. In the lower left frame (d), both water and a TBC segment (arrows) are captured in flight due to the energetic detachment of the TBC. The water droplet can then be found in the next frame at rest on the table top ( $\boldsymbol{e}$, arrow). After rinsing and drying, very little TBC remained bonded to the sample (not shown).

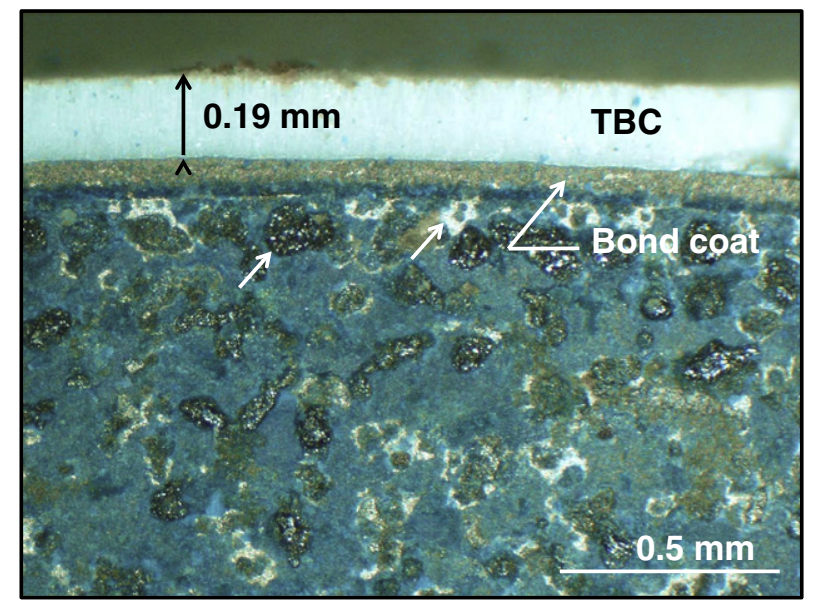

Fig. 10. Optical micrograph of cut edge of sample B2 after $5 \mathrm{hr}$ oxidation at $1150^{\circ} \mathrm{C}$. Intact $\mathrm{TBC}$, lightly oxidized bond coat, dark $\mathrm{NiO}$ nodules and bright bare metal spalling (white arrows) on cut superalloy edge.

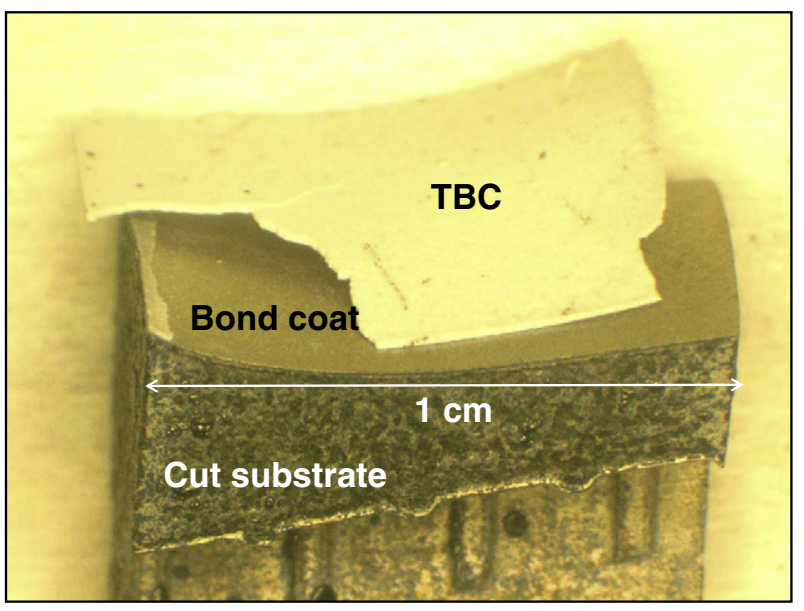

Fig. 11. Optical macrograph of Sample C2 after complete TBC delamination from water drop test following $5 \mathrm{hr}$ intermittent oxidation at $1200{ }^{\circ} \mathrm{C}$.

Of the five videos obtained so far, the time interval between the application of moisture and the first sign of TBC spallation ranged from 1 second to nearly 3 minutes, Table 1 . However, once spallation began, the interval for completion was only 1 to 7 seconds. Indeed the spallation events appeared instantaneous in many regards. The longer intervals were associated with partial spalling and additional incubation time before the next spallation event for the adjacent coating. The sequence of spallation in each video was similar in that failure would start at one edge or corner and progress across the sample. There was no apparent correlation of either of these times with oxidation temperature or time.

While the overall exposed bond coat appearance is that of clean reflective metal, closer examination reveals additional detail. Fig. 14 is an optical surface micrograph of sample $\mathrm{C} 2$, oxidized at $1200^{\circ} \mathrm{C}$ for $5 \mathrm{hr}$. failing by water immersion. It shows a series of entrained scale in more or less a grid-like network of stringers.

\subsection{SEM analysis of failed interfaces}

The surfaces exposed by TBC delamination were examined by SEM/EDS. Fig. 15 shows a typical area of the underside of a spalled fragment of coating after water drop failure for sample B4 oxidized at $1150{ }^{\circ} \mathrm{C}$ for $35 \mathrm{hr}$. For the most part, the TBC fragment retained the thermally grown alumina scale exhibiting an undulating or rumpled

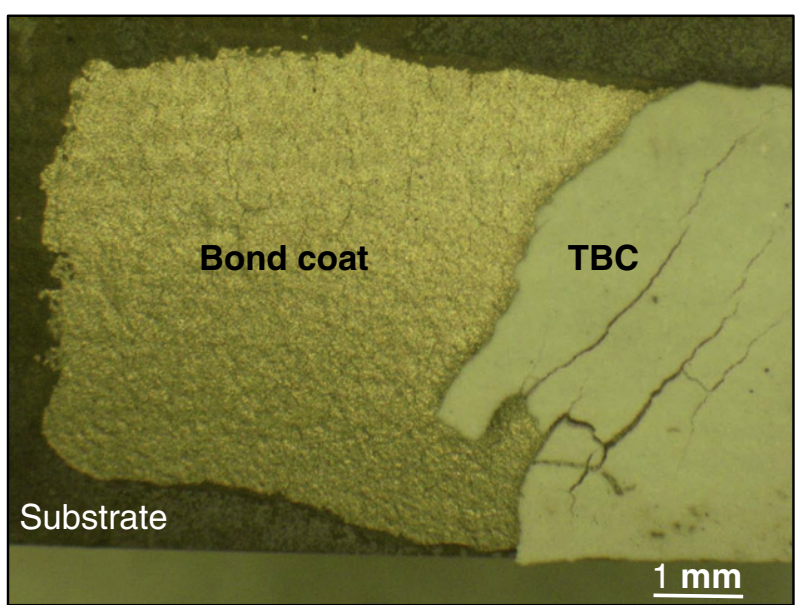

Fig. 12. Optical macrograph of Sample A2. Partial TBC delamination resulting from water drop test following $10 \mathrm{hr}$ intermittent oxidation at $1100^{\circ} \mathrm{C}$. Reflective bare metal bond coat revealed under delaminated TBC and multiply-cracked, but retained, TBC region. 

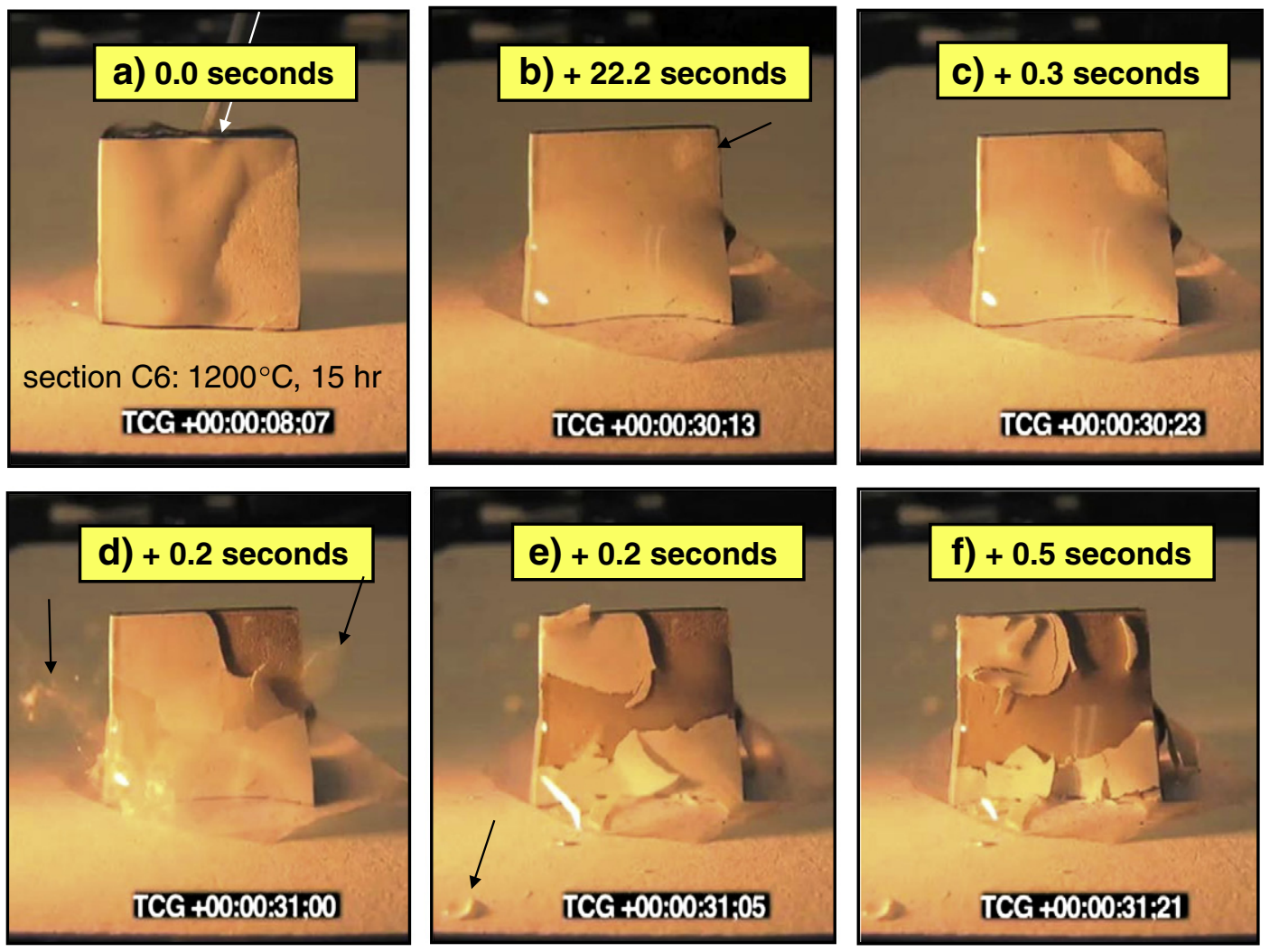

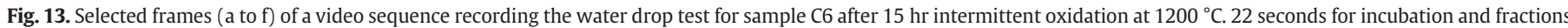
of a second for successive delamination. Arrows show crack initiation, water droplet and coating in flight, and water drop at rest.

morphology (15a). The grain morphology of the scale underside is apparent (15b) with most grains being in a 1-2 $\mu \mathrm{m}$ range. Some highlighted areas in backscatter imaging were shown by EDS to be $\mathrm{Ni}$, Ta- rich oxide or Ni metal alloy, but was alumina overall (15c). Attempts were made to examine the attached alumina scale in fracture sections of spalled TBC fragments, Fig. 16. Unfortunately, no areas of the fracture edges with retained scale were found, even though EDS identified the alumina scale away from the fracture section. It is seen that the spalled TBC coating was about $140 \mu \mathrm{m}$ thick here.

At the exposed side of the blade, much bare metal was revealed with an array of included alumina stringers, Fig. 17, spaced about $0.2-$ $0.5 \mathrm{~mm}$. These stringers are seen to consist of multiple layers of scale that are somewhat wrinkled, distorted and porous, Fig. 18. They also show numerous patches of bright particles found by EDS to be Ta or $\mathrm{Zr}$-rich oxide. At higher magnification, the alumina stringers are seen to be highly convoluted lamellae and not dense. The layered duplex grain morphologies indicate multiple growth sequences, perhaps triggered by cyclic exposures, rumpling, and local detachment of the scale, but without removal by spallation. Finally, the oxide grain imprints, Fig. 19, are decorated with lower Al content lenticular

\section{Table 1}

Video-recorded incubation and propagation times for water drop TBC delamination after various oxidation treatments.

\begin{tabular}{llllll}
\hline Sample & Oxidation & & & \multicolumn{2}{l}{ Spallation (seconds) } \\
\cline { 2 - 3 } \cline { 5 - 6 } & Temperature & Hours & & Incubation & Propogation \\
\hline B1 & $1150{ }^{\circ} \mathrm{C}$ & 12.5 & & 1 & 2 \\
B4 & & 30 & & 163 & 1 \\
C1 & $1200{ }^{\circ} \mathrm{C}$ & 10 & & 2 & 9 \\
C6-a & & 15 & & 22 & 2 \\
C6-b & & 20 & & 3 & 6 \\
\hline
\end{tabular}

precipitates, presumably an Al-depleted Ni-rich phase. Other bright areas, scattered sporadically over the bond coat surface, were noticeably Ta-rich.

\section{Discussion}

The results show that a typical TBC on an actual turbine blade can be made to fail by accelerated oxidation and moisture exposure. While water was used in this test, there is much precedent to show that delayed failure of alumina-metal systems exhibit delayed failure in ambient humidity. This includes the "desktop spallation" delayed failure phenomenon specific to TBCs. Significant scatter can be expected. Indeed

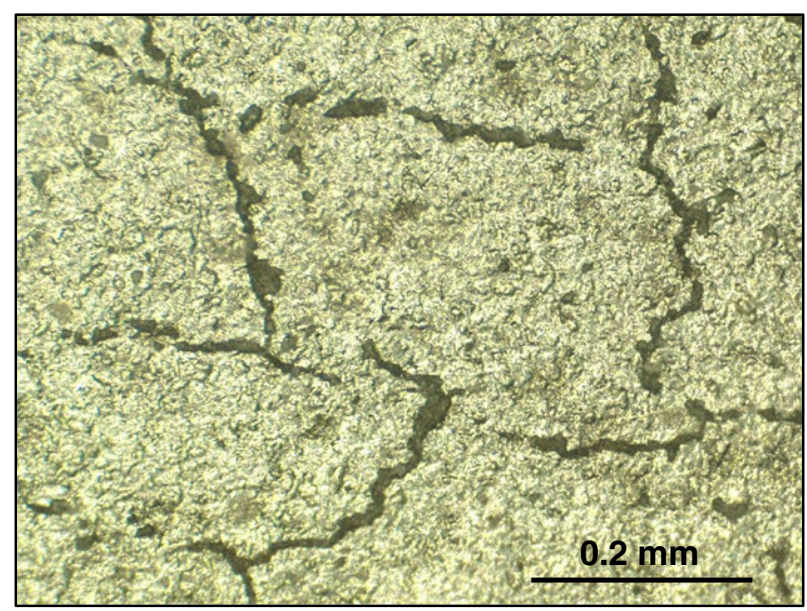

Fig. 14. Optical macrograph of Sample C2 after TBC delamination resulting from a 2 minute water drop test following $5 \mathrm{hr}$ intermittent oxidation at $1200{ }^{\circ} \mathrm{C}$. Reflective bare metal bond coat and network of retained oxide stringers. 

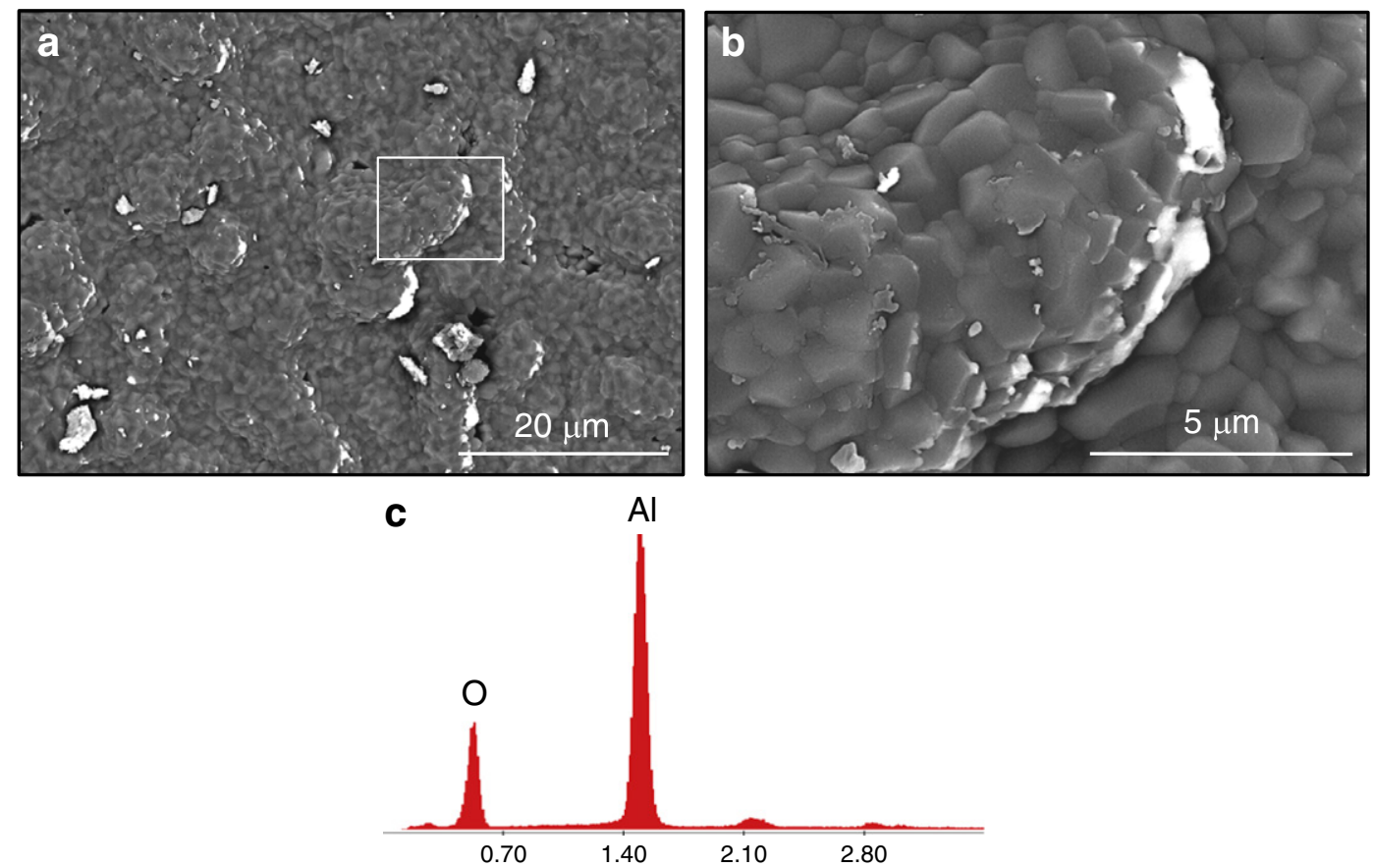

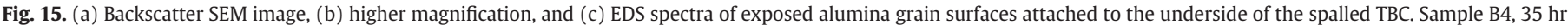
intermittent oxidation at $1150{ }^{\circ} \mathrm{C}$.

there is an element of luck involved with capturing a video of a water drop failure, as failure may occur first on cooldown or may not occur for many more hours of testing. Be that as it may, there does appear to be a strong temperature effect on moisture induced failure, reaching 75 $100 \mathrm{hr}$ or more at $1100^{\circ} \mathrm{C}, 15-35 \mathrm{hr}$. at $1150^{\circ} \mathrm{C}$, and $5-20 \mathrm{hr}$ at $1200^{\circ} \mathrm{C}$. This attests to the increase in stored strain energy in the scale from both the increase in scale thickness with temperature at a given time, as well as the increase in the delta temperature operative for CTE mismatch stresses. Of course the CTE mismatch stress is experienced even on the first cycle, so scale thickening and progressive damage are seen as necessary factors for failure.

In that regard, it is helpful to compare these results with previous studies. A similar coating was tested on test discs and survived 2801 -hr cycles at $1150{ }^{\circ} \mathrm{C}$ before failing by water drop testing after 300 cycles [7]. Other similar coatings failed between 220 and $440 \mathrm{hr}$. In a definitive, extensive study of EB-PVD coatings on a $\mathrm{Ni}(\mathrm{Pt}) \mathrm{Al}$ bond coat, it was found that $5 \mu \mathrm{m}$ of alumina scale was needed to be grown before moisture effects were noted [10]. This required at least 170 cycles (15 min. heat up $+45 \mathrm{~min}$. hot) to $1150^{\circ} \mathrm{C}$. They further showed that failure can be put off

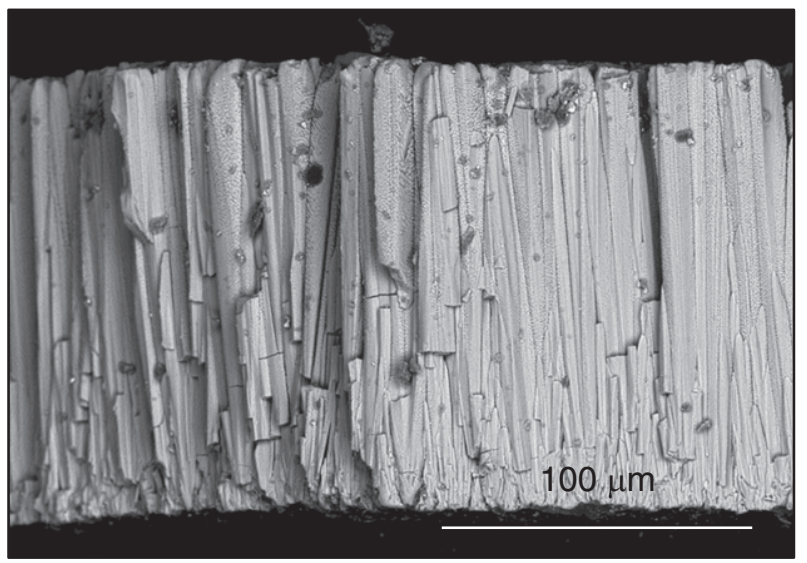

Fig. 16. Backscatter SEM image of a fracture edge of the spalled TBC. Sample B4, $35 \mathrm{hr}$ intermittent oxidation at $1150{ }^{\circ} \mathrm{C}$. by retaining cooled specimens in low humidity, then forced to fail by exposure to ambient humidity or water. Thus, in general, the cut blade sections in the present study appeared to fail at a small fraction of the lives of new coated test buttons. One factor would certainly be the prior engine exposure. Another important consideration is any damage introduced by sectioning and exposing cut interfaces to the oxidation exposure. Other geometric factors may come in to play as well, as the highly curved sections labeled \#1-3 generally failed before the flatter regions in sections \#4-6.

Given that bare reflective metal is exposed upon the water induced failure (and cooling failures), we can conclude again that the oxidemetal interface is the sensitive affected location. The rapidity and explosiveness of the spallation process reflects the release of high levels of stored strain energy in the scale. Incubation times may be a few seconds to a few minutes, but, once started, the delamination process was complete in 1-2 seconds. These observations are similar

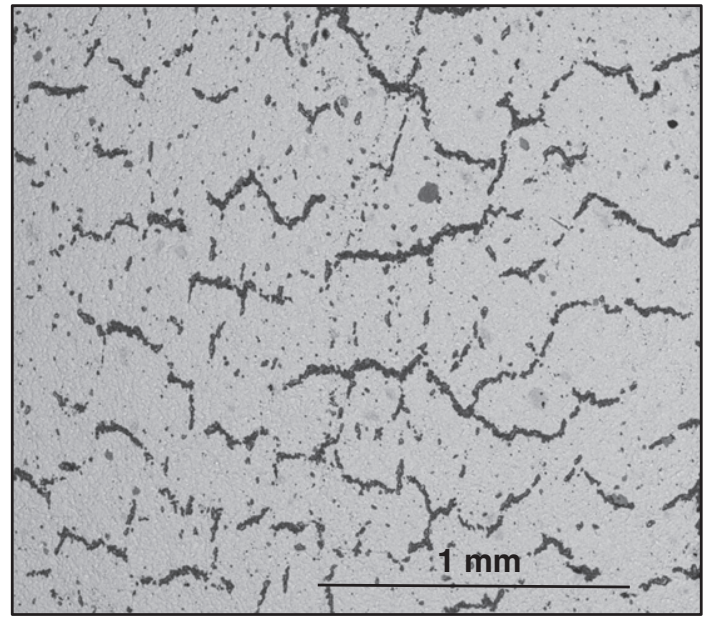

Fig. 17. Low magnification backscatter SEM image of the exposed metal surface showing a network of entrained alumina scale stringers. Sample B4, $35 \mathrm{hr}$ intermittent oxidation at $1150{ }^{\circ} \mathrm{C}$. 

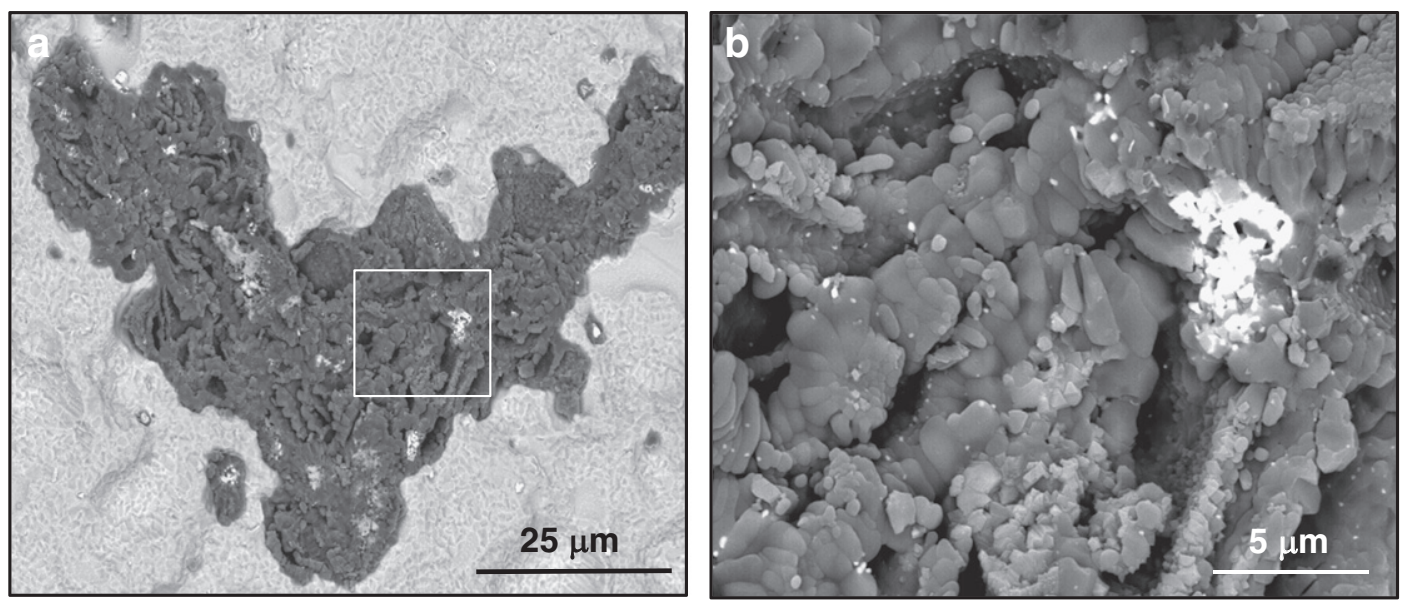

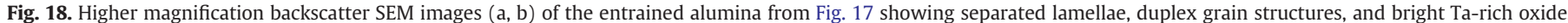
Sample B4, $35 \mathrm{hr}$ intermittent oxidation at $1150^{\circ} \mathrm{C}$.

to those reported in [7-10]. Given the working hypothesis that hydrogen embrittlement is the root cause, this feature may be difficult to rationalize with diffusion times required for hydrogen to transit from one initiation site to the other side of the sample. There seems to be an autocatalytic detachment triggered as soon as new interface is exposed. This may attest to rapid chemical transitions once $\mathrm{Al}$ in the metal is exposed to water molecules. It may also indicate that the interface was partially compromised before the detachment front arrived. For example, moisture may have already penetrated all parts of the entire TBC and began to access the scale-metal interface through cracks in the scale.

The observation of dispersed Ta- and Ti-rich particles in the alumina stringers is evidence of further oxidative degradation, but is not considered to be critical. Though $\mathrm{TiO}_{2}$ is often associated with accelerated scale growth, there is not enough present here to control total interfacial delamination. Ta-rich oxides, on the other hand, often appear as fine, innocuous $\mathrm{NiTa}_{2} \mathrm{O}_{4}$ precipitates, and are correlated with the excellent oxidation resistance of 2 nd generation single crystal alloys [11].

It should also be recalled that sometimes the TBC detaches as one intact layer at one extreme, other times as small segments, or somewhere in between. To some extent, this may be a function of how well the TBC is sintered across growth columns. For DTS of plasma sprayed TBCs, delamination is almost always as a single large piece because of the stronger bonding within splats. These coatings also produce much less spalling to bare metal, leaving much of the alumina scale still attached. This may be because of a less intimate contact of the TBC with the scale as compared to EB PVD TBCs. For scales without a TBC, MIDS is on a much finer level, generally exhibiting spalling on a microscale for highly stressed but moderately bonded scales. Spalling initiates from previously spalled regions or discontinuities in the complex scale microstructure produced by cyclic oxidation. Scale delamination as an intact layer is generally reserved for alloys with poor scale adhesion, i.e., high sulfur levels and no dopants.

One might also envision some aspect of adsorbed droplet surface tension to be affecting interfacial strength. Along these lines, other liquids (acetone, then 200 proof alcohol) were used prior to water immersion after a number of exposures at $1100{ }^{\circ} \mathrm{C}$. However they were never successful in initiating TBC delamination. (Only some slight weight loss resulted from removal of complex oxides from the cut edges of superalloy.)

In review, the observations in the present study are consistent with the prior studies showing moisture-induced DTS [5-10]. As proven by Deneux et al., [10] a minimum amount of pre-oxidation (i.e., scale thickness) is required to sensitize the system to moistureinduced spallation. DTS may be delayed indefinitely if suitably preoxidized coatings are sequestered from moisture. Re-introduction of moisture then causes failure at various incubation times inversely
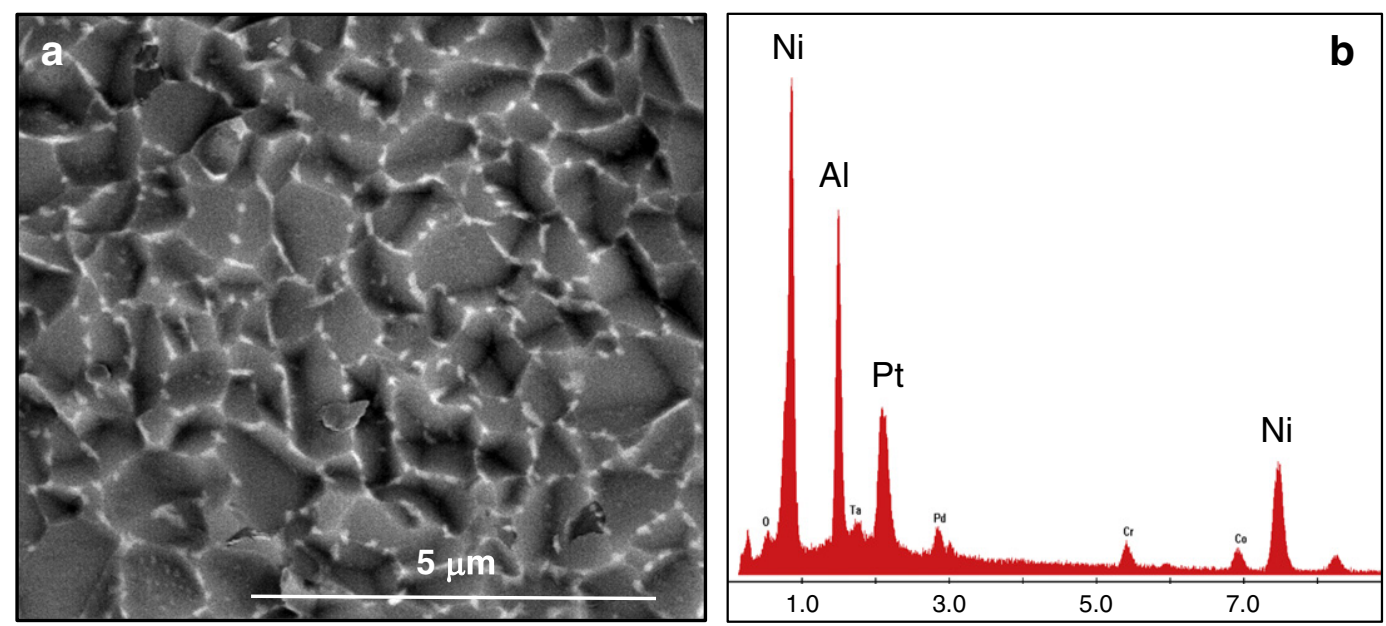

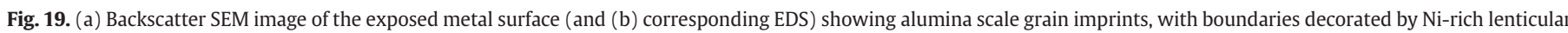
particles. Sample B4, $35 \mathrm{hr}$ intermittent oxidation at $1150{ }^{\circ} \mathrm{C}$. 
related to the level of moisture (e.g., ambient vs water drop or immersion).

The atomistic details of the failure process are still a matter of study and conjecture and are beyond the scope of the present study. Other supporting circumstantial evidence, derived from a number of tangentially related fields, has been presented in a more detailed position paper for the purpose of discussion [1]. These points can be briefly summarized as:

- Hydrogen gas is produced by reaction of water and Al powders.

- $\mathrm{H}$ was detected at the scale-metal interface of anodized Al.

- $\mathrm{Ab}$ initio studies report a $90 \%$ decrease in $\mathrm{Co}-\mathrm{Al}_{2} \mathrm{O}_{3}$ interfacial strength due to $\mathrm{H}$.

- $\mathrm{H}_{2}$ gas, moist air and $\mathrm{Ar}$, and electrochemical $\mathrm{H}$-charging all reduce the ductility of $\mathrm{Ni}_{3} \mathrm{Al}, \mathrm{Fe}_{3} \mathrm{Al}, \mathrm{FeAl}$, and Alloy 718 compared to dry air or vacuum.

- Cathodic H-charging of pure Ni increases the fracture area of intergranular embrittlement from $2 \%$ up to $95 \%$, in combination with segregated sulfur.

- Exposure to moisture or $\mathrm{H}$ and $\mathrm{D}$-implantation produces bubbles and film delamination for native alumina films on $\mathrm{Al}$. (He-implantation does not).

These findings all support moisture-induced interfacial weakening for alumina-Ni(Al) systems. Here we have discussed the general elements of DTS in the context of MIDS [12], in agreement with the conclusions stated in [10]. That is to say, hydrogen 'embrittlement' had been proposed as the mechanism fundamental to both.

While interfacial hydrogen detection is a difficult task [13], the existence of subsurface cracks in the bond coat after water exposure provides indirect but very significant corroborating evidence [10]. Perhaps the suggestion that Ni-rich metal particles adhered to the detached alumina scale in the present work may be a result of some bond coat cracking. Alternatively, hydrogen charging by cathodic polarization was sufficient to detach mature alumina scales that had already been shown to survive water immersion $[2,3]$.

Thus the various chemical factors in play for alumina scale adhesion may now include hydrogen, in addition to sulfur and reactive elements. Though not discussed at length in the present study, previous work suggests that hydrogen may work in concert with sulfur segregation, even at low levels, in reducing scale adhesion. While the present set of experiments may not be directly related to performance in service, it does emphasize the importance of moisture to our understanding of alumina scale adhesion and interpretation of cyclic oxidation under ambient humidity. This factor is usually not considered when performing normal cyclic life tests to failure. The observed failure time may be affected by the frequency and duration of exposure to true ambient room conditions on cool down, which can be shown to have much higher relative humidity than the cooling environment in many cyclic tests [14]. It also raises an interesting question and concept, such as how much life extension is produced by avoiding moisture entirely or by possibly eliminating sensitivity to moisture or hydrogen.

Regarding high temperature tests in moist environments, it has recently been shown that moisture increases surface rumpling of NiPtAl bond coats and decreases TBC life accordingly [15]. No effect was reported regarding delayed spallation, possibly because all failures had already taken place in the furnace environment. The failure locus was again at the scale-bond coat interface, so this interface may still be subject to some of the considerations discussed above. Interdiffused Pt-only bond coats exhibit no rumpling and are consequently immune to furnace moisture content. The higher creep strength of a Pt-modified $\gamma-\gamma$ superalloy compared to that of a $\beta$-phase aluminide is the likely explanation for the differences. However the reason that moisture influences NiPtAl rumpling is not immediately clear.

\section{Summary and Concluding Remarks}

This study has again shown that moisture may induce TBC failure at room temperature, consistent with desktop TBC spallation phenomena for samples cycled near their oxidative limits. It has used cut sections of a turbine blade retired from service and thus relates to actual engine hardware, but in extreme or accelerated tests. A strong temperature dependence was shown, producing water immersion or water drop failures at a maximum of 100,35 , and $20 \mathrm{hr}$ for interrupted oxidation tests at $1100{ }^{\circ} \mathrm{C}, 1150{ }^{\circ} \mathrm{C}$, and $1200{ }^{\circ} \mathrm{C}$, respectively. Sample video recordings of the failure process showed that incubation times of 1 second to 3 minutes may be needed, but then completed in a matter of seconds. The failure surface was unequivocally at the scale-metal interface for vast areas of the sample. After TBC spallation, the reflective metal of the bond coat was easily observed and confirmed by subsequent SEM of the metal, showing imprints of the oxide grains and EDS spectra for the $\mathrm{Ni}(\mathrm{Pt}, \mathrm{Ta}) \mathrm{Al}$ interdiffused bond coat. A network of alumina stringers in the metal was suggestive of biaxial strains, perhaps related to rumpling or craze cracking of the bond coat. The mating side of the spalled TBC was universally covered with alumina. However fracture cross sections were not immediately productive for measuring scale thickness. The results, in total, are consistent with all prior DTS studies. They suggest that moisture exposures play a role in TBC failure after cooling because of detachment of the alumina scale from the bond coat. If these humidity/moisture exposures vary in level, frequency, or duration, they may result in corresponding (uncontrolled) variations in lifetimes as measured in laboratory tests.

\section{Acknowledgements}

The supply of engine hardware and advice of the turbine materials scientists are greatly appreciated. The assistance of Dr. Anita Garg and Michael D. Cuy is gratefully acknowledged for SEM/EDS and video recording the water drop spallation tests, respectively.

\section{References}

[1] J.L. Smialek, "Moisture-Induced Alumina Scale Spallation: The Hydrogen Factor," NASA/ TM-2010-216260, April 201031 pages., http://gltrs.grc.nasa.gov/reports/ 2010/TM-2010-216260.pdf.

[2] J.L. Smialek, JOM, 1, 29-36 (Jan., 2006). Also "Moisture-Induced Delayed Spallation and Interfacial Hydrogen Embrittlement of Alumina Scales," NASA TM 2005-214030, Dec. 200529 pages, http://gltrs.grc.nasa.gov/reports/2005/TM2005-214030.pdf.

[3] J.L. Smialek, Electrochim. Acta 56 (2011) 1823.

[4] V. Sergo, D.R. Clarke, J. Am. Ceram. Soc. 81 (12) (1998) 142.

[5] J.L. Smialek, Ceram. Eng. Sci. Proc. 23 (4) (2002) 485.

[6] J.L. Smialek, J. Therm. Spray Technol. 13 (2004) 66.

[7] J.L. Smialek, Zhu Dongming, Michael D. Cuy, Scr. Mater. 59 (2008) 67.

[8] M. Rudolphi, D. Renusch, M. Schütze, Scr. Mater. 59 (2008) 255.

[9] M. Rudolphi, D. Renusch, H.-E. Zschau, M. Schütze, Mater. Sci. Forum 595-598 (2008) 177

[10] V. Déneux, Y. Cadoret, S. Hervier, D. Monceau, Oxid. Met. 73 (1-2) (2010), doi: 10.1007/s11085-009-9170-1.

[11] Smialek, C.A. Barrett, J.C. Schaeffer, Design for Properties, ASM Handbook, 20, George A. Dieter, Chairman, ASM, Materials Park, OH, 1997, p. 589.

[12] J.L. Smialek, Mater. Sci. Forum, 595-598 (2008) 191-198. (Also NASA TM 2008215206. June 2008, 9 pages; http://gltrs.grc.nasa.gov/reports/2008/TM-2008215206.pdf).

[13] H. Zschau, M. Dietrich, D. Renusch, M. Schütze, J. Meijer, H. Becker, Nucl. Instrum. Methods Phys. Res., Sect. B 249 (2005) 381.

[14] J.L. Smialek, Oxid. Met. 72 (5-6) (2009) 259.

[15] B.A. Pint, G.W. Garner, T.M. Lowe, J.A. Haynes, and Y. Zhang, ICMCTF, (2011) this conference proceedings. 